\title{
An Expressive Avatar for Instant Messaging Endowed with Emotional Intelligence
}

\author{
Alena Neviarouskaya ${ }^{1}$, Helmut Prendinger ${ }^{2}$, and Mitsuru Ishizuka ${ }^{1}$ \\ ${ }^{1}$ University of Tokyo, Department of Information and Communication Engineering, Japan \\ lena@mi.ci.i.u-tokyo.ac.jp, ishizuka@i.u-tokyo.ac.jp \\ ${ }^{2}$ National Institute of Informatics, Japan \\ helmut@nii.ac.jp
}

\begin{abstract}
In this paper, we propose to endow a graphical representation of a user in Instant Messaging - an avatar - with the ability to recognize and to express emotions and to play social nonverbal behaviour, on the basis of textual affect sensing and interpretation of communicative functions conveyed by online conversations. The developed Affect Analysis Model integrated with Instant Messaging (IM) media supports the recognition of affect from text to ensure avatar animation in an appropriate and expressive manner.
\end{abstract}

Keywords: Affect sensing from text, affective user interface, animations, avatar.

Recently, a wide variety of approaches to visualizing the affective content of documents can be observed. The underlying idea is to associate each emotion with a particular pattern of expressive reaction. It has been demonstrated that affect can be represented by means of color [3], expressive text [2,5], static or animated emoticons, cartoon-like characters or human-like avatars, and images of human faces.

In our research, a 2D avatar was designed to automatically represent a user's affect and social nonverbal behaviour in an Instant Messaging (IM) system. Animations of avatar expressive patterns are driven by the output of the developed Affect Analysis Model [4], which is capable of recognizing and interpreting the conveyed emotions and communicative behaviour from not only correctly written text, but also informal messages written in abbreviated or expressive manner. The proposed rule-based approach to affect sensing from text at a sentence-level is described in [4]. For affect categorization, we use the subset of emotional states defined by Izard [1]: 'anger', 'disgust', 'fear', 'guilt', 'interest', 'joy', 'sadness', 'shame', and 'surprise'. As to communicative functions, 'greeting', 'thanks', 'posing a question', 'congratulation', and 'farewell' form the basis for communicative behaviour identification.

After the dominant emotion of the sentence is determined (according to the highest intensity in the resulting emotion vector) and communicative functions are detected, the relevant parameters are sent to the animation engine, which decides the sequence of animations and their duration depending on sentence length, intensity of dominant emotion, personality type of a user, and 'overall mood' of a conversation.

Emotional states of avatar were designed conformably to the relevant expressive means. For example, 'fear' is characterized by: widely open eyes; raised eyebrows; 
open mouth with crooked lips; trembling chin; hands in front of the body quasi pushing the source of fear away. In our work, we consider one personality trait, extraversion, to differentiate ways and strength of avatar expressiveness. In order to enable the avatar to display emotions of different intensity degrees, animations were created according to low, middle, and high level of emotion intensity.

As examples, facial expressions of 'joy', 'anger' and 'fear' of the female avatar designed by us are shown in Fig. 1.

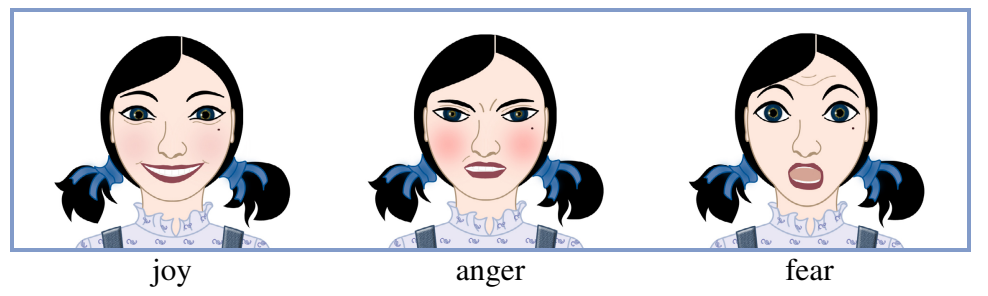

Fig. 1. Examples of emotions displayed by female avatar

The richness of the information cues is a salient aspect of effective interpersonal communication. This holds true also within the virtual environment context. Based on their automatic identification from text, our avatar can act out the five communicative functions listed above. They can be displayed in sequence with emotional animations. However, the communicative function 'posing a question' is an exceptional case. Each of the five communicative functions has an associated specific lexicon and nonverbal behaviour. For example, relevant lexicon and expressive means for 'congratulation' are, respectively: 1) "congratulation", "praiseworthy", gj [good job], llta [lots, lots of thunderous applause], $\mathrm{d}=\left({ }^{\wedge} \mathrm{O}^{\wedge}\right)=\mathrm{b}$ [thumbs up] etc.; 2 ) admiring gaze, smile, clap of the hands. The animations of our avatar also include idle movements so as to provide a sense of liveliness: eye blinks; changing of gaze direction to avoid "dull stare"; yawning; playing with fingers; smoothing hair; folding arms, etc.

In our work, we strive to provide vivid and expressive visual signals to enhance socially oriented online communication media through the visualization of affective states and nonverbal behaviour of an avatar on the basis of textual affect sensing.

\section{References}

1. Izard, C.E.: Human Emotions. Plenum Press, New York, NY (1977)

2. Kalra, A., Karahalios, K.: TextTone: Expressing Emotion Through Text. In: Costabile, M.F., Paternó, F. (eds.) INTERACT 2005. LNCS, vol. 3585, pp. 966-969. Springer, Heidelberg (2005)

3. Liu, H., Selker, T., Lieberman, H.: Visualizing the Affective Structure of a Text Document. In: Proceedings of the Conference on Human Factors in Computing Systems, CHI'03, pp. 740-741 (2003)

4. Neviarouskaya, A., Prendinger, H., Ishizuka, M.: Textual Affect Sensing for Sociable and Expressive Online Communication. In: Proceedings of the 2nd International Conference on Affective Computing and Intelligent Interaction, Springer, Heidelberg (2007)

5. Strapparava, C., Valitutti, A., Stock, O.: Dances with Words. In: Proceedings of the International Joint Conference on Artificial Intelligence, India, pp. 1719-1724 (2007) 